10-1-2021

\title{
Understanding community health volunteer incentive preferences in Kenya
}

Frontline Health Project

Johns Hopkins Bloomberg School of Public Health

Follow this and additional works at: https://knowledgecommons.popcouncil.org/departments_sbsr-rh

Part of the Health Services Research Commons, and the Public Health Education and Promotion Commons

How does access to this work benefit you? Let us know!

\section{Recommended Citation}

Frontline Health Project and Johns Hopkins Bloomberg School of Public Health. 2021. "Understanding community health volunteer incentive preferences in Kenya," Results brief. Washington, DC: Population Council. 


\section{Understanding Community Health Volunteer Incentive Preferences in Kenya}

\section{BACKGROUND}

Community health workers (CHWs) are critical actors within community health systems, providing a range of reproductive, maternal, child health information, counseling, and services. $\mathrm{CHWs}$ facilitate access to primary healthcare (PHC) services and serve as trusted intermediaries between communities and other skilled health workers in countries such as Kenya. This is crucial as CHWs help to address issues that the formal sector is not always equipped to tackle such as access to basic health services including health education (1-3).

In Kenya, community health services (CHS) are implemented through community health units (CHUs), each serving a population of 5,000 people. Community health volunteers ( $\mathrm{CHVs}$ ) who serve these units are chosen by the community and trained by community health extension workers (CHEWs). CHVs offer a range of community-based information and services including encouraging antenatal care, facility-based deliveries, postnatal care for mother and newborn, family planning, malaria prevention, testing for HIV, nutrition, and safe water, sanitation, and hygiene practices $(5,6)$.

Despite their evidence of effectiveness, $\mathrm{CHVs}$ are considered as volunteer health workers and remuneration of $\mathrm{CHVs}$ varies by county in Kenya. CHVs are often tasked with demanding daily activities and incentives assist in improving work motivation. As recommended by the $2018 \mathrm{WHO}$ guideline on health policy and systems support to optimize CHW programs, financial remuneration as well as non-financial incentives are critical for improving $\mathrm{CHV}$ performance and retention (7).

Stated preference elicitation methods such as discrete choice experiments (DCEs) can help better understand incentive preferences and tradeoffs CHVs are willing to make (8). This study utilized a DCE to understand incentive preferences of $\mathrm{CHVs}$ with the aim of improving motivation, performance, and retention of $\mathrm{CHVs}$.

This brief summarizes qualitative and quantitative findings from the Frontline Health project's DCE study in Kenya, implemented

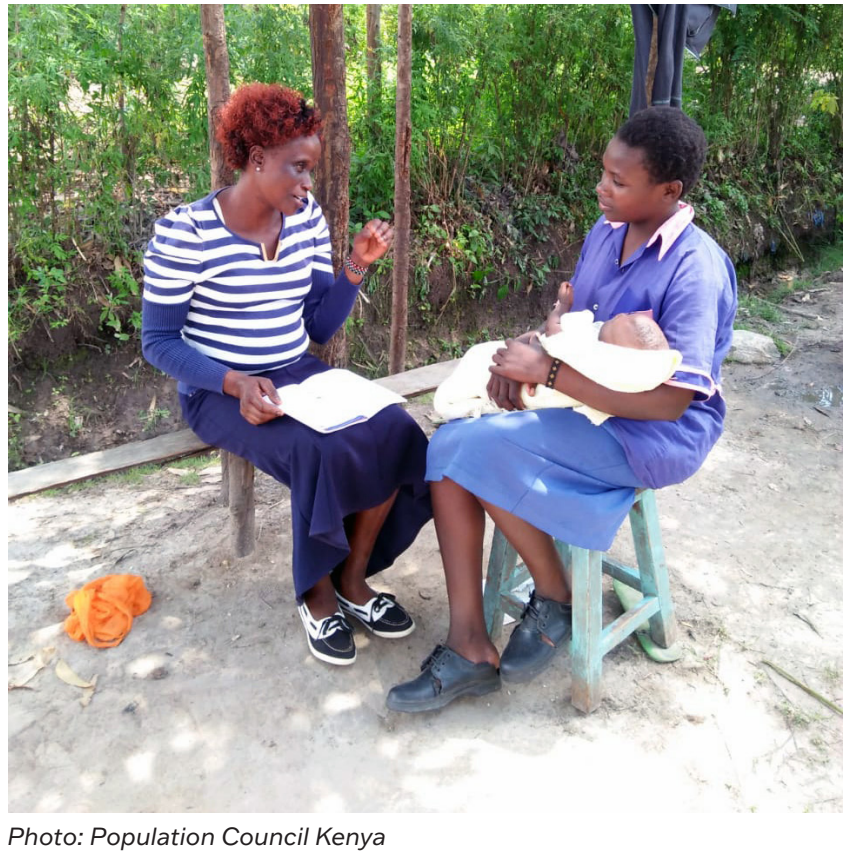

KEY FINDINGS

1. CHVs in Kenya prefer incentive packages that combine both monetary and non-monetary incentives to serve as work motivators.

2. Incentive packages for CHVs must be context dependent even within the same country in order to maximize impact.

3. Transportation was the most important attribute for CHVs followed by tools of trade, monthly stipends, and income generating activities. 


\section{ABBREVIATED METHODS}

The study was conducted among conducted among $211 \mathrm{CHVs}$ from two sub-counties of Kilifi ( $n=109)$, a rural coastal county, and Bungoma $(n=102)$, a western rural agrarian county. Implementation occurred in two phases in 2019. In phase one, financial and non-financial attributes that influence CHV performance were identified through the use of focus group discussions (FGDs) with both $\mathrm{CHVs}$ and their supervisors (CHEWs). In-depth interviews (IDIs) were conducted on the national level with policymakers and key organizations that implement CHS to understand their views on $\mathrm{CHV}$ incentive preferences. Phase two utilized a cross-sectional quantitative DCE survey among CHVs to further elicit their incentive preferences.

with collaborators from the Johns Hopkins Bloomberg School of Public Health and Pathfinder International, and supported by Kenya's Ministry of Health.

\section{RESULTS}

\section{MONETARY INCENTIVES PLAY A VARIED ROLE IN CHW MOTIVATION}

\section{Monthly Stipends}

CHVs gave importance to monthly stipends however there were geographical variations to their importance in Kenya. For example, importance varied when considering the importance of monthly stipends, transport, and tools of trade. Kilifi County considered monthly stipends as least important while CHVs in Bungoma rated them as the second most important attribute.

\section{NON-MONETARY INCENTIVES PLAY A KEY ROLE IN CHW MOTIVATION}

\section{Transportation}

Lack of transportation methods for $\mathrm{CHVs}$ is reported as a factor limiting $\mathrm{CHV}$ work performance
$(9,10)$. CHVs had a positive preference for jobs that provided both motorcycles and bicycles to reach $\mathrm{CHUs}$ as opposed to those that only provided bicycles. In the DCE, motorcycles were the preferred transportation vehicle as they can also be used communally as a source of income when not being utilized for serving patients.

\section{Availability of Tools}

CHVs preferred increased availability of reporting tools and job aids. When tools and jobaids are replenished regularly, they facilitate accurate reporting, quickly disseminate updates, and improve CHV performance.

\section{Workload}

CHVs found that workloads were high specifically in the context of utilizing paper tools. CHVs are sometimes unable to engage in personal activities or responsibilities when tasked with reporting monthly household visits across large areas (11).

\section{Official Identification}

CHVs described the provision of identification materials such as shirts, badges, caps, branded bags and reflector jackets as useful for carrying out daily tasks and increasing community trust.

\section{BOX 1: PHASE 2 DCE RESULTS}

CHVs viewed improved transportation mechanisms as the most important attribute for improving work performance followed by tools of trade and monthly stipends. Recognition on the community level was discussed as a key motivator that will enhance their work.

Amongst the least important attributes, monthly stipends were not found to be as important because among those reporting receipt of financial compensation, less than $15 \%$ received amounts that were within the recommended range of KES 2000 (US \$20) per month. Approximately 40\% received KES 5000 (US \$50) per month. The least important incentive package attribute was income generating activities. 
Sources of identification were described as being particularly useful during the rainy seasons to maintain the quality of training materials. $\mathrm{CHVs}$ were willing to accept a KES 2514 (US \$25.14) reduction in monthly stipend for an incentive package that provided identification.

\section{Work Recognition}

CHVs preferred incentives that provide recognition at the community level as opposed to those that provide recognition at the facility level, or those that only provide award mechanisms for the best performing $\mathrm{CHV}$. Since $\mathrm{CHVs}$ are often believed to be vehicles for facilitating community agency and triggering social change (12), recognition at the community level is central to maintaining trust between communities and $\mathrm{CHVs}$.

\section{BOX 2: STUDY STRENGTHS AND LIMITATIONS}

This study provides useful information for policymakers on the attributes of a successful incentive package for CHVs in Kenya and other similar contexts. However, since DCEs are a stated preference approach, they are prone to bias as they present hypothetical alternatives to respondents. Furthermore, this DCE adopted a forced-choice elicitation format (lack of opt-out) which might have exacerbated hypothetical bias. Nonetheless, qualitative methods were used to identify and validate incentive attributes which may have reduced hypothetical bias. Although these results show heterogeneity in terms of incentive preferences for $\mathrm{CHVs}$ in different locations, they are valuable as they illustrate the importance of creating adaptable context dependent incentives for resource limited settings.

\section{CONCLUSION AND RECOMMENDATIONS}

Creating incentive packages that fit the needs of $\mathrm{CHVs}$ plays a key role in improving $\mathrm{CHV}$ work performance and community health outcomes. This study found that a bundled incentive of both financial and non-financial packages is necessary to provide a conducive working environment for CHVs.

This study's findings have significant health policy implications through highlighting the need for increased attention on creating CHV incentive structures which combine an effective transport system for $\mathrm{CHVs}$, essential tools of trade to enable them to perform their roles, and adequate monthly stipends. Key CHV program stakeholders in Kenya should consider the importance of the above attributes to facilitate CHV satisfaction and performance.

We recommend that policymakers and key organizations implement a combination of context dependent monetary and non-monetary incentives to improve $\mathrm{CHW}$ motivation and job performance, thus paving the way for improved health outcomes.

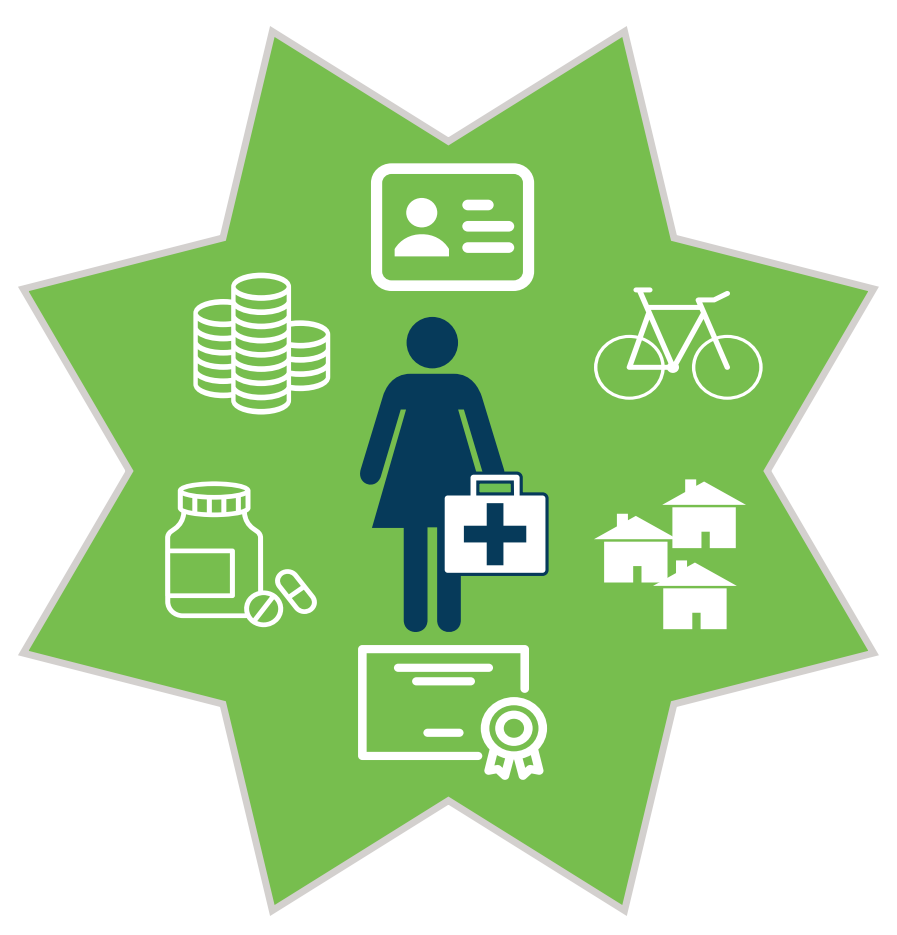




\section{RESEARCHER CONTACTS}

Smisha Agarwal

Assistant Professor, Department of International Health The Johns Hopkins Bloomberg School of Public Health sagarw23@jhu.edu

Timothy Abuya

Population Council, Kenya

tabuya@popcouncil.org

Suggested citation: Frontline Health Project and Johns Hopkins Bloomberg School of Public Health. 2021. Understanding Community Health Volunteer Incentive Preferences in Kenya. Washington, D.C.: Population Council.

\section{REFERENCES}

1. Perry HB, Zulliger R, Rogers MM. Community health workers in low-, middle-, and high-income countries: an overview of their history, recent evolution, and current effectiveness. Annu Rev Public Health. 2014;35:399-421.

2. O'Donovan J, O'Donovan C, Kuhn I, Sachs SE, Winters N. Ongoing training of community health workers in low-income andmiddle-income countries: a systematic scoping review of the literature. BMJ Open. 2018;8(4):e021467.

3. Agarwal S, Anaba U, Abuya T, Kintu R, Casseus $A$, Hossain $S$, et al. Understanding incentive preferences of community health workers using discrete choice experiments: a multicountry protocol for Kenya, Uganda, Bangladesh and Haiti. BMJ Open. 2019;9(12):e033601.

4. MoH. Guidelines on Continued Provision of Community Health Services in the Context of Corona Virus Pandemic in Kenya. 2020.
5. Wangalwa G, Cudjoe B, Wamalwa D, Machira Y, Ofware P, Ndirangu M, et al. Effectiveness of Kenya's Community Health Strategy in delivering communitybased maternal and newborn health care in Busia County, Kenya: non-randomized pre-test post test study. Pan Afr Med J. 2012;13 Suppl 1:12.

6. MoH. Evaluation report of the Community Health Strategy implementation in Kenya. 2010.

7. Ayandipo O, Wone I, Kenu E, Fasehun LK, Ayandipo $\mathrm{O}$, Gaye F, et al. Cancer ecosystem assessment in West Africa: health systems gaps to prevent and control cancers in three countries: Ghana, Nigeria and Senegal. Pan Afr Med J. 2020;35:90.

8. Hensher DA, Rose JM, WH G. Applied Choice Analysis. Cambridge: Cambridge University Press; 2015.

9. Kok MC, Dieleman M, Taegtmeyer M, Broerse JE, Kane $\mathrm{SS}$, Ormel $\mathrm{H}$, et al. Which intervention design factors influence performance of community health workers in low- and middle-income countries? A systematic review. Health Policy Plan. 2015;30(9):1207-27.

10. Brunie A, Wamala-Mucheri P, Otterness C, Akol $A$, Chen M, Bufumbo L, et al. Keeping community health workers in Uganda motivated: key challenges, facilitators, and preferred program inputs. Glob Health Sci Pract. 2014;2(1):103-16.

11. Aseyo RE, Mumma J, Scott K, Nelima D, Davis $\mathrm{E}$, Baker KK, et al. Realities and experiences of community health volunteers as agents for behaviour change: evidence from an informal urban settlement in Kisumu, Kenya. Hum Resour Health. 2018;16(1):53.

12. Kok MC, Broerse JEW, Theobald S, Ormel H, Dieleman M, Taegtmeyer M. Performance of community health workers: situating their intermediary position within complex adaptive health systems. Hum Resour Health. 2017;15(1):59. 